\title{
Prevalencia de patología cervical premaligna y maligna en pacientes con citología negativa
}

\author{
José Vicente Erazo*
}

RESUMEN: En el Departamento de Patología del Hospital Universitario San José de Popayán, se procesaron durante el año de 1992 un total de $\mathbf{1 5 . 4 8 0}$ citologías de las cuales sólo el $\mathbf{0 . 9 5 \%}$ fueron positivas (PAP III-IV-V). El 93\% correspondió a PAP II de la "Antigua Clasificación" (gráfica 1). Con el fin de incrementar el porcentaje tan bajo de patología cervical premaligna y maligna con el uso rutinario de la citología, se determinó llevar a cabo un monitoreo cervical utilizando: test de Schiller, colposcopia y biopsia dirigida a las pacientes con citología negativa (PAP II) y que a la especuloscopia presentaran ectropión (erosión) cervical, parcial o total. El análisis histopatológico de 124 pacientes con estas características demostró que un 59.6\% tenían patología cervical premaligna o maligna: Lesión Intraepitelial escamosa de bajo grado (L.SIL) 51.6\%; lesión intraepitelial escamosa de alto grado (H. SIL) 6.47\% y Carcinoma INVASOR 1.67\%. Para el tratamiento de estos pacientes se tuvo en cuenta: su edad, paridad, deseos de reproducción, la histopatología y la posibilidad de seguimiento.

PALABRAS CLAVES: Patología cervical, colposcopia, ectropión.

L-SIL (Lesión Intraepitelial Escamosa de bajo grado)

H-SIL (Lesión Intraepitelial Escamosa de alto grado)

\section{Introducción}

Colombia en su condición de país pobre, presenta una de las mayores incidencias de morbilidad y mortalidad por carcinoma de cérvix a nivel mundial (1). En el Hospital Universitario San José de Popayán, las tasas de CARCINOMA INVASOR en comparación con la neoplasia intraepitelial cervical (NIC: I-II-III), han descendido año tras año, desde un $98 \%$ para el año 1963, hasta llegar a un $19.44 \%$ en 1993 (2) lo cual demuestra una tasa ideal comparable con la de los países desarrollados (Gráfica 2).

La patología cervical maligna y premaligna de cérvix puede ser detectada mediante el uso rutinario de la citología exo-endocervical, utilizando buena técnica en la toma de la muestra, en la fijación y en su interpretación. Sin embargo este método presenta índices de falsos negativos que oscilan entre el 5 y $55 \%$ según diferentes autores $(3-11,18)$.

Grandes controversias a nivel mundial se han presentado en cuanto a la diversidad de técnicas utilizadas en mejorar la calidad de la muestra citológica como: aspirado endocervical, raspado exocervical exhaustivo, espátula de Ayre, Citobrush, aplicación de la biología molecular HVP-DNA (18-22) como marcador oncogénico, citometría de flujo, cervicografía, reestructuración de la clasificación citológica (Sistema de Bethesda) (21), etc., con el fin de disminuir las altas tasas de FALSOS NEGATIVOS (12-15).

* Médico Gineco Obstetra, Hospital Universitario San José, Popayán.
Gráfica 1

CITOLOGIA EXO-ENDOCER VICAL

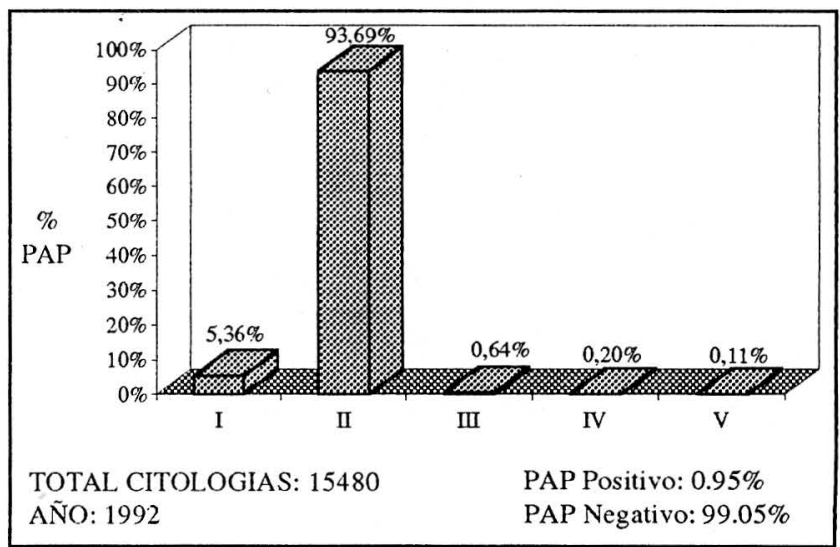

Gráfica 2

CA INVASOR - VS- NIC: I-II-III. ENDOCERVICAL




El presente estudio prospectivo analiza 124 casos de pacientes que reunieron las siguientes características: Haber tenido un resultado de citología exo-endocervical negativo, en el último año o historia de varias citologías negativas y presentar a la especuloscopia; algún grado parcial o total de ectroprión cervical.

\section{Definición de términos}

1. Citología negativa. Teniendo en cuenta la clasificación antigua: son los PAP I y II con o sin atipias.

2. Ectropión. Presencia exocervical de la mucosa endocervical sobre un estroma fibrovascular de tipo inflamatorio (Blaustein's: Patology of the female genital tract "Third Edition").

3. Colposcopia

- Normal. Si los hallazgos son compatibles con una zona de transformación típica o con cambios inflamatorios.

- Anormal. Si los hallazgos de la zona de transformación son atípicos como: epitelio acetoblanco, micropapilar, leucoplasia, mosaico, vasos atípicos; según recomendaciones de la Federación Internacional de Patología Cervical y Colposcopia (IFCPC). Séptimo Congreso Mundial, Roma Mayo de 1990 (17).

\section{Histopatología}

- Lesión Intraepitelial Escamosa de Bajo Grado (LSIL), donde se incluyen cuadros de patología referentes a Papilomavirus Humano (HVP), Displasia Leve o NIC-I.

- Lesión Intraepitelial Escamosa de Alto Grado (HSIL) que incluye dentro del mismo parámetro los cuadros histopatológicos de: displasia moderada y severa o NIC: II-III y el carcinoma In Situ (11-20).

\section{Material y métodos}

Se realizó un estudio prospectivo y analítico de 124 pacientes que acudieron a la Unidad de Patología Cervical del Hospital Universitario San José de Popayán y que presentaban los siguientes criterios de selección:

1. Una o más citologías negativas para neoplasia (PAP II con o sin atipias).

2. Cérvix erosionado (ectropión) a la especuloscopia.

En forma metodológica a cada una de las pacientes se les practicó:

a. Especuloscopia

b. Colposcopia

c. Test de ácido acético al 5\%

d. Test de Schiller

e. Biopsia dirigida por colposcopia para estudio histopatológico

Una vez obtenido el reporte histopatológico, se siguió una conducta de manejo individual dependiendo de la edad, paridad, extensión de la lesión, factibilidad en seguimiento para cada una de las pacientes estudiadas (Tabla 3 ).

\section{Resultados}

Se procesa manualmente la información de 124 casos de citología exo-endocervical negativa (PAP II) de las pacientes que acudieron a la Unidad de Patología Cervical de nuestro hospital, analizando las siguientes variantes:

\section{Edad}

En nuestra casuística el problema de la patología cervical no respeta edades, encontrándose presente antes de los 20 años como después de los 40 (Gráfica 3).

\section{Gráfica 3}

PATOLOGIA CERVICAL Y GRUPOS DE EDAD

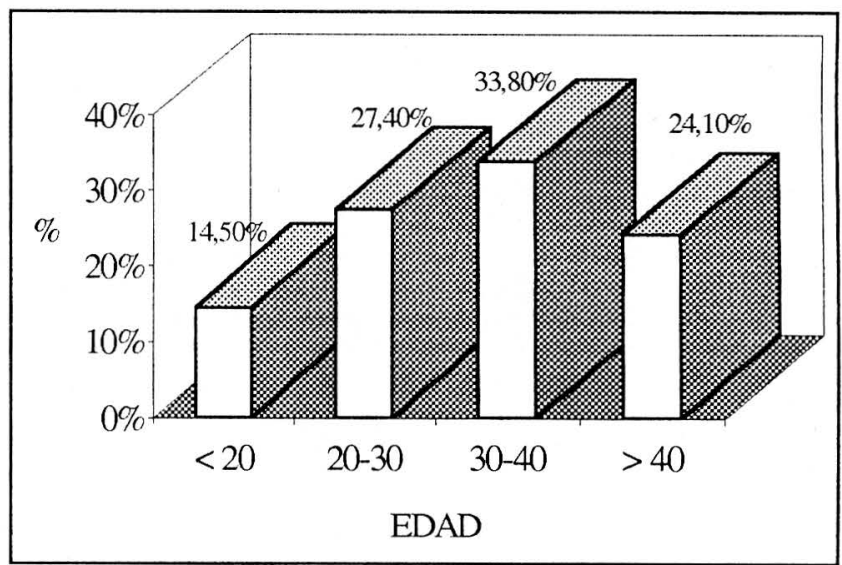

\section{Paridad}

En cuanto a la paridad tampoco hubo significancia estadística, no existiendo diferencias significativas entre primíparas y grandes multíparas (Gráfica 4).

\section{PATOLOGIA CERVICAL Y PARIDAD}

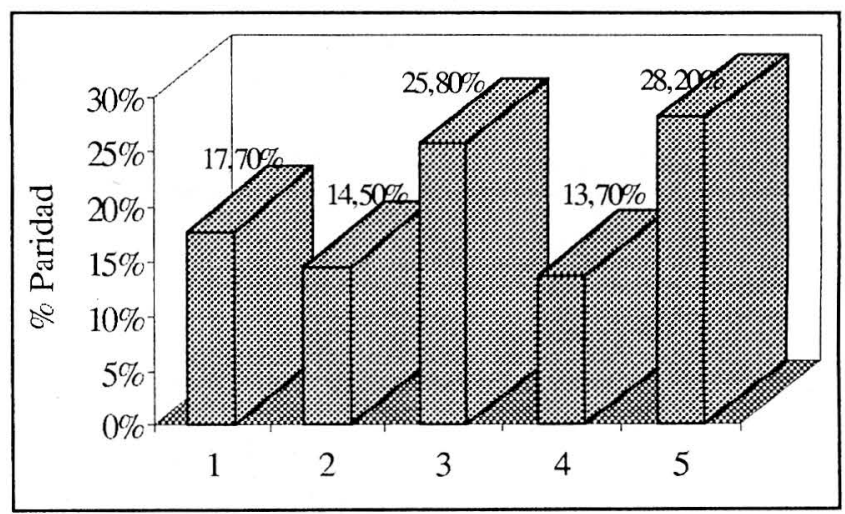

\section{Colposcopia}

La colposcopia es una técnica de diagnóstico subclínico de aceptación mundial para el estudio de pacientes con citología normal o anormal. La correlación colposcopia e histopatología en nuestro estudio dio resultados excelen- 
tes ante hallazgos colposcópicos anormales en un $71.77 \%$, se pudo comprobar por Histopatología-patología premaligna y maligna en un $59.6 \%$ de los casos (Tabla 1 ).

Tabla 1

HALLAZGOS COLPOSCOPICOS DE LAS

\section{MUJERES CON CITOLOGIA EXO-ENDOCERVICAL NEGATIVA (PAP II)}

(Según terminología y clasificación recomendada por la Federación Internacional de Patología Cervical (IFCPC) y Colposcopia en el 7o. Congreso Mundial de Roma $1990^{(17)}$ )

\begin{tabular}{|c|c|c|}
\hline Colposcopia & $\begin{array}{c}\text { No. } \\
\text { Pacientes }\end{array}$ & Porcentaje \\
\hline $\begin{array}{l}\text { Hallazgos colposcópicos } \\
\text { normales } \\
\text { Hallazgos colposcópicos } \\
\text { anormales } \\
\text { 1. Epitelio aceto-blanco } \\
\text { 2. Micropapilas } \\
\text { 3. Leucoplasia } \\
\text { 4. Mosaico } \\
\text { 5. Vasos atípicos }\end{array}$ & $\begin{array}{l}35 \\
89\end{array}$ & $\begin{array}{l}25.22 \\
71.77\end{array}$ \\
\hline Total & 124 & 100 \\
\hline
\end{tabular}

Test del ácido acético 124 casos positivos

Test de Schiller 124 casos positivos

\section{Histopatología}

Los hallazgos histopatológicos de las biopsias dirigidas por colposcopia de 124 pacientes con citología negativa (PAP II), alcanzaron altos índices de prevalencia de patología cervical $59.6 \%$ incluyendo en este grupo dos casos $(1.6 \%)$ de carcinoma invasor (Tabla 2 ).

\section{Tabla 2}

RESULTADOS HISTOPATOLOGICOS DE 124 PACIENTES CON CITOLOGIA NEGATIVA Y ECTROPION CERVICAL

\begin{tabular}{|c|c|c|}
\hline Histopatología & $\begin{array}{c}\text { No. } \\
\text { Pacientes }\end{array}$ & Porcentaje \\
\hline $\begin{array}{l}\text { 1. Cervicitis-endocervicitis } \\
\text { crónica o aguda }\end{array}$ & 44 & 35.4 \\
\hline 2. Pólipo endocervical & 6 & 4.8 \\
\hline $\begin{array}{l}\text { 3. L-SIL (HPV-NIC-I) } \\
\text { 4. H-SIL } \\
\text { (NIC-II-III-Ca in situ) }\end{array}$ & $\begin{array}{l}64 \\
8\end{array}$ & $\begin{array}{l}51.6 \\
\text { cervical } \\
\text { premaligna } \\
=58 \%\end{array}$ \\
\hline $\begin{array}{l}\text { 5. Ca invasor } \\
\text { •Adenocarcinoma } \\
\text { •Ca. escamoso }\end{array}$ & $\begin{array}{l}1 \\
1\end{array}$ & $1.6 \rightarrow \begin{array}{l}\text { Patología } \\
\text { cervical } \\
\text { maligna }= \\
16 \%\end{array}$ \\
\hline Total & 124 & 100 \\
\hline
\end{tabular}

\section{Tratamiento}

Fue totalmente individualizado para cada paciente, teniendo en cuenta los protocolos de manejo del Instituto Nacional de Cancerología (16), en relación con el componente histopatologico, edad, paridad y posibilidad de seguimiento (Gráfica 5).

Gráfica 5

TRATAMIENTOS DE 124 PACIENTES SEGUN RESULTADO HISTOPATOLOGICO

\begin{tabular}{|lcc|}
\hline Tratamiento & No. de casos & Porcentaje \\
\hline 1. Electrocauterio & 46 & 37.09 \\
2. Crioterapia & 42 & 33.8 \\
3. Radio frecuencia & 12 & 9.6 \\
4. Conización Qx & 16 & 12.6 \\
5. Histerectomía & 6 & 4.8 \\
6. Radioterapia & 2 & 1.6 \\
\hline Total & 124 & 100 \\
\hline
\end{tabular}

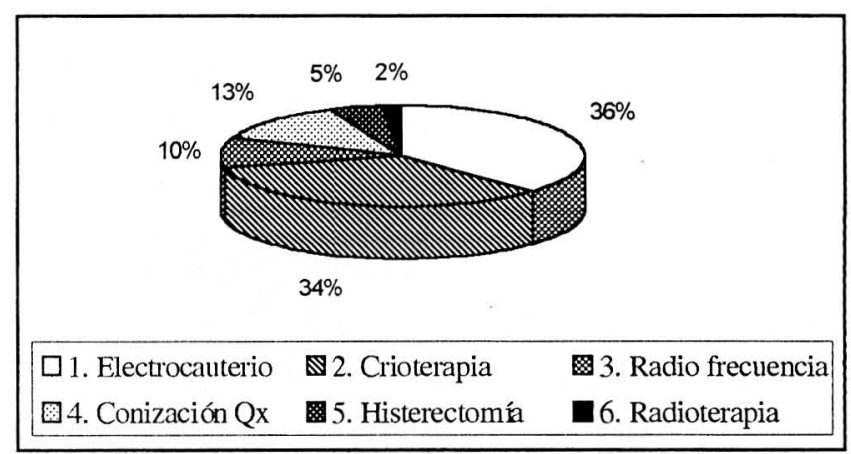

\section{Conclusiones}

- La citología exo-endocervical es extremadamente sensible y específica en detectar cáncer y un buen porcentaje de las lesiones escamosas intraepiteliales de alto grado (H-SIL).

- Es poco sensible en detectar lesiones escamosas intraepiteliales de bajo grado (L-SIL) como: Papilomavirus Humano (HPV) o displasia leve (NIC I).

- El hallazgo de un cuello normal es poco frecuente, ante la alta incidencia de Ectropion sobre todo en mujeres que han tenido uno o más partos. En nuestro medio el Ectropion es una lesión que precede a la neoplasia intraepitelial en un alto porcentaje.

- El test de Schiller, sencillo y de costo insignificante es de mucha utilidad en la toma de la biopsia cervical, en las regiones donde no es posible el uso rutinario de la colposcopia.

- El monitoreo rutinario de cérvix sobre todo en pacientes de alto riesgo, donde se incluya: especuloscopia, Test de Schiller, test de ácido-acético, colposcopia, biop- 
sia dirigida, etc. y en un futuro complementar el estudio con anticuerpos monoclonales para HPV, serían las metas ideales a pesar de su costo en clasificar un cérvix como Normal o Anormal.
- En nuestro estudio casi el $60 \%$ de las pacientes con citología negativa (PAP II) y utilizando dos factores de riesgo: parto y Ectropion cervical, concomitaron con patología premaligna o maligna.

\section{BIBLIOGRAFIA}

1. Correa P. and Llanos. Morbility and Mortality from Cancer en Cali. Colombia J. Natl, Cancer Inst. 1966; 36: 17-745.

2. Hospital Universitario San José de Popayán. Laboratorio de Citopatología. Estadística, 1993.

3. Koss Leopold MD. The Papanicolau Test for Cervical Cancer Detection. A Triumph and Tragedy. Jama 1989; 261: 737-743.

4. Andrew MD., Miya Sawac MD. The Significate of Negative Papanicolau Smear. Symposioum Contemporary, Ob-Gyn 1989.

5. Averette H., MD. Management for atipical PAP Smear. Symposioum Contemporary, Ob-Gyn 1989.

6. Fetherson WC. La Citología Falso-Negativa y el Cáncer Invasor de Cérvix. Clin. Obst. Ginecol. 1984; 4: 1133.

7. Feldman MJ., Seeve CC. False Positive Cervical Cytology An Important Reason for Colposcopy. Am. J. Obst. Gynec. 1977; 129-141.

8. Noumott JS. Atipia in Cervical Cytology as a Risk for Intraepithelial Neoplasia. Am. J. Obst. Gynec. 1987; 156: 628.

9. Jones Ped et al. Evaluation of the atipical PAP Smears. Am. J. Obst. Gynec. 1987; 157-544.

10. Fetherson WC. False Negative Cytology in Invasive Cancer or the Cervix. Clin. Obst-Gyn 1983; 26: 929.

11. Davis GL., Hernández E. Atipical Squamous cells in Papanicolau Smear. Obst. Gynecol. 1987; 69: 43.

12. Spitzen M., Krumboltz BA., Seltzer LA. Cervical Cancer detected by Cervicography in a Patient with Negative Cervical Cytology. Obst. Ginecol. 1986; 68: 685.
13. Vermund Sh et al. Molecular Diagnosis of genital Papilomavirus Infection. Am. J. Obst.-Gynecol. 1989; 160: 304-308.

14. National Cancer Institute Works Shop. The 1988 Bethesda system for reporting Cervical-Vaginal Cytologic Diagnosis. Jama 1989; 262: 931-934.

15. Boon ME et al. Analysis of Five Sampling Methods for the Preparation of Cervical Smear. Acta Cyto 1988; 33: 843-848.

16. Instituto Nacional de Cancerología. Detención y Control del Cáncer de Cuello Uterino. Manual de Técnicas y Procedimientos. Santafé de Bogotá. 1991.

17. Staf A., MD and Wilbank G. International Terminology of Colposcopy Obst. Gynecol. 1991; 313-315.

18. Pairwaits. False-Negative Papanicolau Smears from women with Cancerous and Precancerous Lesions of the Uterine Cervix. Acta Cytol 1991; 35: 40-46.

19. Kenneth D., Hatch. Preinvasive Cervical Neoplasia. Seminar in Oncology 1994; 21: 12-26.

20. Richart RM. Clinical Comentary: A modified Terminology for Cervical Intraepitelial Neoplasia. Obst.-Gynecol 1990; 75: 131-133.

21. Sheperd John. Prevential cervical cancer. The role of the Bethesda System. American Family Physician 1995; 51-434-439.

22. Symposium, HPV DNA Testing comes of age, Ralph M. Richart MD. Moderator. Contemporary Ob/Gyn 1995; 40: 79-99.
- SERVICIO DE CORREO CERTIFICADO - SERVICIO DE CERTIFICADO ESPECIAL - ENCOMIENDAS CONTRA REEMBOLSO - SERVICIO TARIFA POSTAL REDUCIDA - SERVICIO DE FILATELIA

- SERVICIO ELECTRONICO

- SERVICIO INTERNACIONAL APR/SAL

- SERVICIO RESPUESTA COMERCIAL

- SERVICIOS ESPECIALES

Teléfonos para quejas y reclamos: 3340304 - 3415536 - Santafé de Bogotá, D.C. - Colombia

Cuente con nosotros hay que creer en los Correos de Colombia 